INPLASY

PROTOCOL

To cite: Zhong et al. Efficacy and Safety of Eptinezumab for migraine: Findings from randomized controlled trials. Inplasy protocol 2021100037. doi:

10.37766/inplasy2021.10.0037

Received: 12 October 2021

Published: 12 October 2021

Corresponding author:

Yi Zhong

yizhong19980628@163.com

Author Affiliation:

Department of Clinical

Medicine, The First Affiliated

Hospital of Soochow

University, Suzhou, Jiangsu

Province, China.

Support: No sources of financial.

Review Stage at time of this submission: Completed but not published.

Conflicts of interest:

None declared.

\section{Efficacy and Safety of Eptinezumab for migraine: Findings from randomized controlled trials}

Review question / Objective: Calcitonin gene-related peptides (CGRP)are considered as a possible preventive treatment for migraine. Eptinezumab, a novel CGRP antagonists, was recently approved for clinical treatment. The aim of this systematic review and meta-analysis is to evaluate the efficacy and safety of eptinezumab administration compared to placebo in migraine patients.

Condition being studied: Eptinezumab, as a new class of CGRP antagonists, has been approved for migraine prevention in adults in 2020. As eptinezumab comes into clinical use, we collected data from a number of randomized controlled trials to perform a meta-analysis. The purpose of this meta-analysis is to shed light on the efficacy and safety of eptinezumab compared with placebo. Simultaneously, a subgroup analysis was completed to evaluate the efficacy and safety of different doses.

INPLASY registration number: This protocol was registered with the International Platform of Registered Systematic Review and Meta-Analysis Protocols (INPLASY) on 12 October 2021 and was last updated on 12 October 2021 (registration number INPLASY2021100037).

\section{INTRODUCTION}

Review question / Objective: Calcitonin gene-related peptides (CGRP)are considered as a possible preventive treatment for migraine. Eptinezumab, a novel CGRP antagonists, was recently approved for clinical treatment. The aim of this systematic review and meta-analysis is to evaluate the efficacy and safety of eptinezumab administration compared to placebo in migraine patients.

Condition being studied: Eptinezumab, as a new class of CGRP antagonists, has been approved for migraine prevention in adults 
in 2020. As eptinezumab comes into clinical use, we collected data from a number of randomized controlled trials to perform a meta-analysis. The purpose of this metaanalysis is to shed light on the efficacy and safety of eptinezumab compared with placebo. Simultaneously, a subgroup analysis was completed to evaluate the efficacy and safety of different doses.

\section{METHODS}

Search strategy: We systematically searched relevant articles in the electronic databases PubMed, EMBASE, Cochrane Library and US National Institutes of Health Clinical Trials Registry in February 2021, using a combination of the following keywords: Eptinezumab or ALD403, and migraine or chronic migraine, no date limitations or language restrictions. In addition, we manually filtered the reference list from RCTS and restricted our systematic search to ensure all studies included in this study are relevant.

Participant or population: A total of 2, 739 patients from 4 multi-centered doubleblinded trials were pooled.

Intervention: The dose of $\mathbf{1 0} \mathbf{~ m g , ~} \mathbf{3 0 ~} \mathrm{mg}, \mathbf{1 0 0}$ $\mathrm{mg}, 300 \mathrm{mg}, 1000 \mathrm{mg}$ Eptinezumab and placebo.

Comparator: Efficacy and safety between placebo and eptinezumab included the change from baseline in monthly migraine days (MMDs) over weeks 1šC12, and the

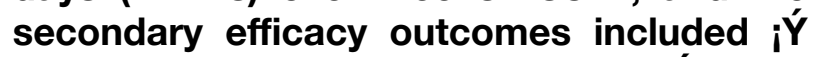
$75 \%$ migraine responder rate, ¡Ý $50 \%$ migraine responder rate and $100 \%$ migraine responder rate. The safety outcome included the proportions of participants who experienced any TEAE and any Serious AE (SAE).

Study designs to be included: We systematically searched relevant articles in the electronic databases PubMed, EMBASE, Cochrane Library and US National Institutes of Health Clinical Trials Registry in February 2021, using a combination of the following keywords: Eptinezumab or ALD403, and migraine or chronic migraine, no date limitations or language restrictions. we used the Review Manager 5.4 software to conduct metaanalysismeta-analysis to compare efficacy and safety of eptinezumab between placebo.

Eligibility criteria: Study will be involved if the inclusion criteria were followed: (a) study type: Randomized Controlled Trial; (b) language restriction: no language restriction; (c) participants: any sex, any ethnicity, aged 18 to 75 years with a diagnosis of migraine at or before 50 years of age, had a history of migraine for 12 months with $\geq 14$ headache days per month. (d) intervention: $10 \mathrm{mg}, \mathbf{3 0} \mathrm{mg}, 100 \mathrm{mg}$, and $300 \mathrm{mg}$ eptinezumab and placebo. We excluded case reports, case reviews, retrospective studies, and studies with populations aged 75 years and older.

Information sources: We systematically searched relevant articles in the electronic databases PubMed, EMBASE, Cochrane Library and US National Institutes of Health Clinical Trials Registry in February 2021, using a combination of the following keywords: Eptinezumab or ALD403, and migraine or chronic migraine, no date limitations or language restrictions.

Main outcome(s): Range from baseline in monthly migraine days (MMDs) over weeks1-12; Three kinds of outcomes were assessed, and all doses of eptinezumab has significant efficacy compared to placebo in terms of three second outcomes, $\geq 75 \%$ migraine responder rate with any dose of eptinezumab, $\geq 50 \%$ migraine responder rate with any dose of eptinezumab, $100 \%$ migraine responder rate with any dose of eptinezumab. Treatment-related adverse events and serious adverse events.

Quality assessment / Risk of bias analysis: We use The Review manager 5.4 software to create the risk of bias plot. The Cochrane collaboration uniform criteria were followed, including: selection bias, performance bias, detection bias, attrition bias, reporting bias, and other potential biases. We conducted a sensitivity test to 
assess the potential bias of these involved four studies. Of these four included studies, all the studies were at low risk of publication bias. For incomplete outcome data and Selective reporting, the Ashina and Silberstein study had an unclear risk of bias, which was shown in detail in Fig 7.

Strategy of data synthesis: In this study, we used the Review Manager 5.4 software to conduct meta-analysis. Mean difference (MD) and relative risk (RR) with their $95 \%$ confidence intervals (Cl) were used to evaluate the efficacy and safety of eptinezumab. Heterogeneity among the trials was assessed by using the Chisquared test and the 12 statistics. We applied Random-effects model to analyze the data. If the $12>50 \%$, the data would be considered as obvious heterogeneity. In addition, we divided the subjects into different subgroups according to the dose and made a subgroup analysis. Details of the subgroup analysis will be described later in the article.

Subgroup analysis: A subgroup analysis of the change from baseline in monthly migraine days (MMDs) among different doses shows that $300 \mathrm{mg}$ has better efficacy, $10 \mathrm{mg}$ (MD = -1.10, 95\% Cl: -2.79, $-0.59, \mathrm{p}=0.20$ ), $30 \mathrm{mg}$ (MD $=-1.42,95 \% \mathrm{Cl}$ : $-2.60,-0.23, p=0.02), 100 \mathrm{mg}$ (MD = -1.55, 95\% Cl: $-2.57,-0.54, \mathrm{p}<0.01), 300 \mathrm{mg}$ (MD $=-2.02,95 \% \mathrm{Cl}:-3.12,-0.93, \mathrm{p}<0.01)$ and $1000 \mathrm{mg}$ (MD $=-1.00,95 \% \mathrm{Cl}:-2.20,0.20, \mathrm{p}$ $<0.01$ ) compared with the placebo. A subgroup analysis of the secondary efficacy outcomes of $\geq 75 \%$ migraine responder rate with $10 \mathrm{mg}(\mathrm{RR}=1.30,95 \%$ $\mathrm{Cl}: 0.82,2.06, \mathrm{p}=0.27), 30 \mathrm{mg}(\mathrm{RR}=1.46$, 95\% Cl: 1.09, 1.95, $\mathrm{p}=0.01), 100 \mathrm{mg}$ (RR = $1.59,95 \%$ Cl: $1.29,1.96, \mathrm{p}<0.01), 300 \mathrm{mg}$ (RR $=1.95,95 \% \mathrm{Cl}: 1.60,2.39, \mathrm{p}<0.01)$ and $1000 \mathrm{mg}$ (RR $=3.75,95 \% \mathrm{Cl}: 1.63,7.81, \mathrm{p}<$ 0.01) compared with the placebo. A subgroup analysis of $10 \mathrm{mg}(R R=1.01$, 95\% Cl: 0.82, 1.26, $p=0.91), 30 \mathrm{mg}(\mathrm{RR}=$ $0.92,95 \% \mathrm{Cl}: 0.77,1.10, \mathrm{p}=0.35), 100 \mathrm{mg}$ (RR = 1.01, 95\% Cl: 0.91, 1.11, p = 0.92), 300 mg (RR $=1.06,95 \% \mathrm{Cl}: 0.96,1.17, \mathrm{p}=0,24)$ and $1000 \mathrm{mg}(\mathrm{RR}=1.08,95 \% \mathrm{Cl}: 0.82,1.43$, $p=0.58$ ) was done to intuitively compare the difference in primary safety outcome between these four doses.

Sensitivity analysis: There is no sensitivity analysis in this review.

Language: No language limits.

Country(ies) involved: China.

Keywords: Eptinezumab; migraine; efficacy; safety;meta-analysis.

Contributions of each author:

Author 1 - Yi Zhong made substantial contributions to the conception and design of the study, acquisition of data, analysis and interpretation of data, and drafted the article.

Email: yizhong19980628@163.com

Author 2 - Jiahe Wang made substantial contributions to the conception and design of the study, acquisition of data, analysis and interpretation of data, and drafted the article.

Email: wangjiahe_96@163.com

Author 3 - Hang $\mathrm{Li}$ revised the article critically for important intellectual content.

Email: neurosurgeryli@163.com

Author 4 - Siyuan Yang revised the article critically for important intellectual content.

Email: 18862166919@139.com

Author 5 - Xiang $\mathrm{Li}$ supervised the data analysis, designed the study, and revised the article.

Email: neurosurgerylee@163.com

Author 6 - Xinmin Zhou supervised the data analysis, designed the study, and revised the article.

Email: jeanne88@163.com

Author 7 - Gang Chen supervised the data analysis, designed the study, and revised the article.

Email: nju_neurosurgery@163.com 